\title{
Human Papilloma Virus in Cervical Cancer - An Institutional Study
}

\author{
Radhika Katragadda, S. Ravikumar* and S. Kirtana \\ Department of Microbiology, Government Medical College, Omandurar Govt. Estate, \\ Chennai, Tamilnadu, India \\ *Corresponding author
}

\section{Keywords \\ Human Papilloma \\ Virus, Cervical \\ carcinoma, High \\ grade Squamous \\ Intraepithelial \\ Lesion}

Article Info

Accepted:

12 May 2019

Available Online:

10 June 2019

\section{A B S T R A C T}

Cervical carcinoma (CA cervix) is the fourth most common cause of death and the second most common cancer in women world-wide. It is a slowly progressing neoplasm that may take 10-20 years to manifest. CA cervix is one of the few vaccine-preventable cancers because it is caused almost exclusively by an exogenous infective agent, the Human Papilloma Virus (HPV). Among the high-risk strains, strains 16, 18, 45, 31, 33, 35, 39, 51, $52,56,58,59$ and 68 are most commonly associated with cervical cancer. Strains 16, 18 and 45 are the most oncogenic, and progress more rapidly to squamous cell carcinomas. Strain 16 is the most prevalent. Strain 18 is more onco-potent and produces cancers that progress faster and have a worse prognosis. This study is aimed to identify the prevalence of particular strains of high-risk HPV associated with cervical cancer in our geographic and demographic niche. Cervical smear sample was collected from 23 women with newly diagnosed cervical cancer attending the out-patient clinic in the Departments of Radiation Oncology and Surgical Oncology. DNA extraction and Polymerase Chain Reaction (PCR) using type-specific primers for both high-risk types of HPV (16/18) was done. Of the two high-risk HPV strains (16 and 18), we found that 56.5\% of patients had HPV strain 16 alone and $13 \%$ had both strains 16 and 18 , while none had only strain 18 . The remaining 7 cases had neither. This study demonstrates the clear association of HPV infection with cervical carcinomas. There was a high correlation between the high-risk HPV strain 16 and invasive cervical carcinoma. The presence of high-risk HPV strains 16 and 18 in $69.5 \%$ of the sample suggests that vaccine prophylaxis would significantly reduce the incidence of CA cervix.

\section{Introduction}

Cervical carcinoma (CA cervix) is the fourth most common cause of death and the second most common cancer in women world-wide (World cancer report, 2014; Faridi et al., 2011). In South India, especially in the northeastern districts of Tamil Nadu, cervical and penile cancers are highly prevalent, with
Chennai having the highest incidence of uterine and cervical cancer in the country (Sreedevi et al., 2015; Berlin Grace et al., 2009). $90 \%$ of cervical cancers are squamous cell carcinomas (SCCs) and the rest are adenocarcinomas, with a negligible number falling under other categories (National cancer institute, 2014). It is a slowly progressing neoplasm that may take $10-20$ 
years to manifest (World cancer report, 2014). Coupled with the frequent lack of resources for diagnosis and treatment in underdeveloped countries, this insidious disease is often detected at advanced stages where remission and survival are low, even with appropriate treatment (Muliira et al., 2017). CA cervix is one of the few vaccine-preventable cancers because it is caused almost exclusively by an exogenous infective agent, the Human Papilloma Virus (HPV) (Bosch et al., 1999; Walboomers et al., 1999). Thus CA cervix can be considered an infective disease, making prophylaxis and prevention of the condition possible.

HPV is a non-enveloped DNA virus that infects and replicates solely in the basal keratinocytes of stratified squamous epithelium (Schiller et al., 2010). It is a sexually transmitted pathogen that gains entry into the deeper layers of epithelium through micro-abrasions produced during intercourse. Once inside a cell, the viral DNA integrates itself with the host genome. The viral DNA consists of three functional coding regions: $\mathrm{E}$ - a gene coding early viral function, L - a gene coding late viral function, and LCR - a long control region which lies between $\mathrm{E}$ and $\mathrm{L}$. The E gene codes for two onco-proteins, E6 and E7, which target and destroy the primary tumor suppressors p53 and RB protein inside the cell. The resulting mitotic dysregulation leads to uncontrolled multiplication of increasingly mutated cells that culminates in CA cervix (Mantovani et al., 2001; Boyer et al., 1996).

Not all strains of HPV are equally oncogenic. Low-risk strains of HPV (such as strains 6 and 11 , or those that belong to the same phylogenetic class) only cause genital warts that never progress to malignancy. Among the high-risk strains, strains $16,18,45,31,33,35$, 39, 51, 52, 56, 58, 59 and 68 are most commonly associated with cervical cancer
(Munoz et al., 2003). Strains 16, 18 and 45 are the most oncogenic, and progress more rapidly to squamous cell carcinomas (Clifford et al., 2003). Strain 16 is the most prevalent. Strain 18 is more onco-potent (Barbosa et al., 1989) and produces cancers that progress faster (Burger et al., 1989) and have a worse prognosis (Nakagawa et al., 1996).

By the age of 30, HPV infection is almost ubiquitous among women. So merely detecting the presence of HPV infection is of little value in the management of CA cervix. Identifying the type of HPV strains associated with cervical cancer is more important in guiding patient management and instituting prophylactic measures against the infection in women who are at risk of developing cervical cancer.

A HPV vaccine has been widely acclaimed as helping prevent development of infections, and consequently, in bringing down the incidence of cervical cancer. The vaccine is highly specific only to certain viral strains, and provides very little cross immunity to other HPV strains. Strain typing can therefore guide vaccine development, and decide the most appropriate version of the vaccine for use in a certain at-risk population. The strains associated with High grade Squamous Intraepithelial Lesion (HSIL) and cancerous lesions vary among different demographics and geographic locations (Ahmadi et al., 2017; Bedoya -Pilozo et al., 2017). Knowledge about the prevalent HPV strains in a region will direct the vaccine policy of that area.

This study aims to study the prevalence of HPV strains 16 and 18 in patients with CA cervix in our tertiary care hospital in the city of Chennai, and observe the prevalence of selected risk factors for cervical cancer in the same population. 


\section{Materials and Methods}

The study population consisted of 23 women with newly diagnosed cervical cancer attending the out-patient clinic in the Departments of Radiation Oncology and Surgical Oncology.

Only untreated patients who had clinically or histopathologically confirmed cervical cancer were included in the study population, after getting their informed consent.

Those patients who were unwilling to participate in the study, or had contraindications to the procedure of collecting samples, were excluded. Those patients who had recurrent tumors after completion of treatment, or who were undergoing radiation or chemotherapy at the time of presentation, were also excluded from the study.

Patient data was collected using a standardized proforma. The procedure for sample collection was explained to the patient.

After a digital vaginal examination to assess the clinical stage of the tumour, a cervical smear sample was collected in the regular manner using a sterile nylon-flocked swab.

The sample, along with the brush portion of the collection device, was immediately placed in Viral Transport Medium and refrigerated at $-20^{\circ}$ Celsius until further processing.

\section{Laboratory analysis}

\section{DNA extraction}

HPV is a cell associated virus. Cells in the sample are lysed to release both HPV DNA (which is the subject of this study) and cellular DNA, which serves as a control for sample adequacy.

\section{PCR amplification and analysis}

Polymerase Chain Reaction (PCR) using type-specific primers for both high-risk types of HPV (16/18), along with the primer for $\beta$ globin (National cancer centre, 2014), was carried out to evaluate samples for the presence and type/strain of HPV DNA PCR was carried out using PCR amplification kits, using type-specific primers from the L1 region for HPV 16 (GCCTGTG TAGGTGTTGAGG and TGGATTTAC TGCAACATTGG), the E1 regions for HPV 1 (GTGGACCAGCAAATACAGGA and TCC AACACGTGGTCGTTGCA), to amplify 246 bp, $162 \mathrm{bp}, 280 \mathrm{bp}$, and $360 \mathrm{bp}$ fragments, respectively.

A reaction mixture of $50 \mu \mathrm{l}$ was prepared using the 1X PCR buffer with $3.5 \mathrm{mM} \mathrm{MgCl}$ $2,1.25 \mathrm{U}$ Taq DNA polymerase enzyme, 200 $\mu \mathrm{M}$ of each of the dNTPs (dATP, dGTP, $\mathrm{dCTP}$, and dTTP), $1 \mu \mathrm{M}$ of each of the primers (Forward and Reverse), and 2-3 $\mu$ g of genomic DNA per $50 \mu 1$ reaction mixture. The reaction mixture without a DNA template, and that with a standard DNA template of known molecular size (provided in the PCR kit itself), were used as negative and positive controls, respectively. Thirty cycles of PCR were carried out for HPV 16/18. The PCR products were stored at $4^{\circ} \mathrm{C}$ for further analysis.

\section{Interpretation and analysis}

The PCR products were analysed by electrophoresis in $2 \%$ agarose gel along with ethidium bromide. A molecular weight marker of 100 bp range was run simultaneously to identify the molecular weight of the PCR products. The DNA bands were visualized by UV trans-illumination and analysed using the gel documentation system for molecular size determination with respect to the DNA marker. 


\section{Statistical analysis}

The data collected from patients was tabulated and analysed to ascertain frequency of prior HPV infection and the prevalence of each strain in the study sample, as well as associations of clinical variables with outcomes. This data was then correlated with the strain typing data obtained from the lab and significance associations (if any) were noted.

\section{Results and Discussion}

There were 23 participants in the study, all of whom had attended the Out-Patient clinic in the Departments of Surgical and Radiation oncology in the months of July-September 2018. All patients had pain with abnormal bleeding or leucorrhea. None of the patients had been previously diagnosed with cervical malignancies or undergone treatment for the same.

All but two patients fell within the ages of 40 to 70 years, with a median age of 55 years. The youngest patient enrolled in the study was 35 years old, and the eldest was $86.39 \%$ of patients were between ages 51 and 60 , while $31 \%$ were between 41 and 50 years old.

All patients came from a low socioeconomic class (as calculated using the modified Kuppuswamy scale) and most were illiterate (according to the UNESCO definition).

They could write their own name, but did not know to read or write, and lacked formal education. Of 3 patients who had formal schooling, 2 had attended primary school, and one had finished middle school.

Many study participants were married within a year of attaining menarche. Of the 23 women, $78 \%$ were married before age 20 , and among them $50 \%$ had married between the ages of 15 and 17 . Only $22 \%$ of the study population married after 18 .

Two out of the study population of 23 patients (9\%) were child marriages, where the bride was under the age of 14. Most women gave birth to their first child within a year of marriage, as the correlation between age at birth of the first child (Chart-5) and marital age (Chart-4) clearly demonstrates

All the women enrolled in the study had children. Most had 2 to 3 full-term pregnancies. $74 \%$ had $2-5$ children, and $17 \%$ had more than 5 children. Only 2 women (9\%) had fewer than 2 children. None of the patients in our study followed sanitary practices during menstruation, and many lacked proper toilet facilities at home. 61\% used either public toilets or practiced open defecation. Only 9 women said their homes had attached bathrooms.

The greater portion of our study group were found to be immune-competent, with none showing evidence of chronic immunosuppression. Only 2 patients (9\%) had diseases like diabetes mellitus that might compromise immunity. None of the study participants were Human Immunodeficiency Virus (HIV) positive, and only one of them (4.5\%) was Hepatitis B Virus (HBV) positive.

Of the 23 women, $18(78 \%)$ presented to hospital for the first time with advanced cancers (stage II or stage III lesions). Only a few patients (13\%) who had undergone regular periodic cancer screening were diagnosed with cervical cancer at an early stage.

$86 \%$ of the observed tumors were Squamous cell carcinomas. Histopathology reports of the remaining $14 \%$ ranged from occasional atypia to severe dysplasia. Adenocarcinomas were not observed in our study sample. 
Of the 23 samples, $13 \quad(56.5 \%)$ were associated with only strain 16 . None of the samples had HPV-18 alone. 3 (13\%) were associated with both HPV-16 and HPV-18. $30.5 \%$ of the samples tested negative for either high-risk HPV strain 16 or 18.

Human Papilloma Virus (HPV) has long been implicated as a causative agent for cervical carcinoma by virtue of its universal association in patients diagnosed with the condition. This study aims to identify the prevalence of particular strains of high-risk HPV associated with cervical cancer in our geographic and demographic niche. Of the two high-risk HPV strains (16 and 18), we found that $56.5 \%$ of patients had HPV strain 16 of alone and $13 \%$ had both strains 16 and 18, while none had only strain 18 . The remaining 7 cases had neither.

Chart.1 Age group of the study population $(n=23)$

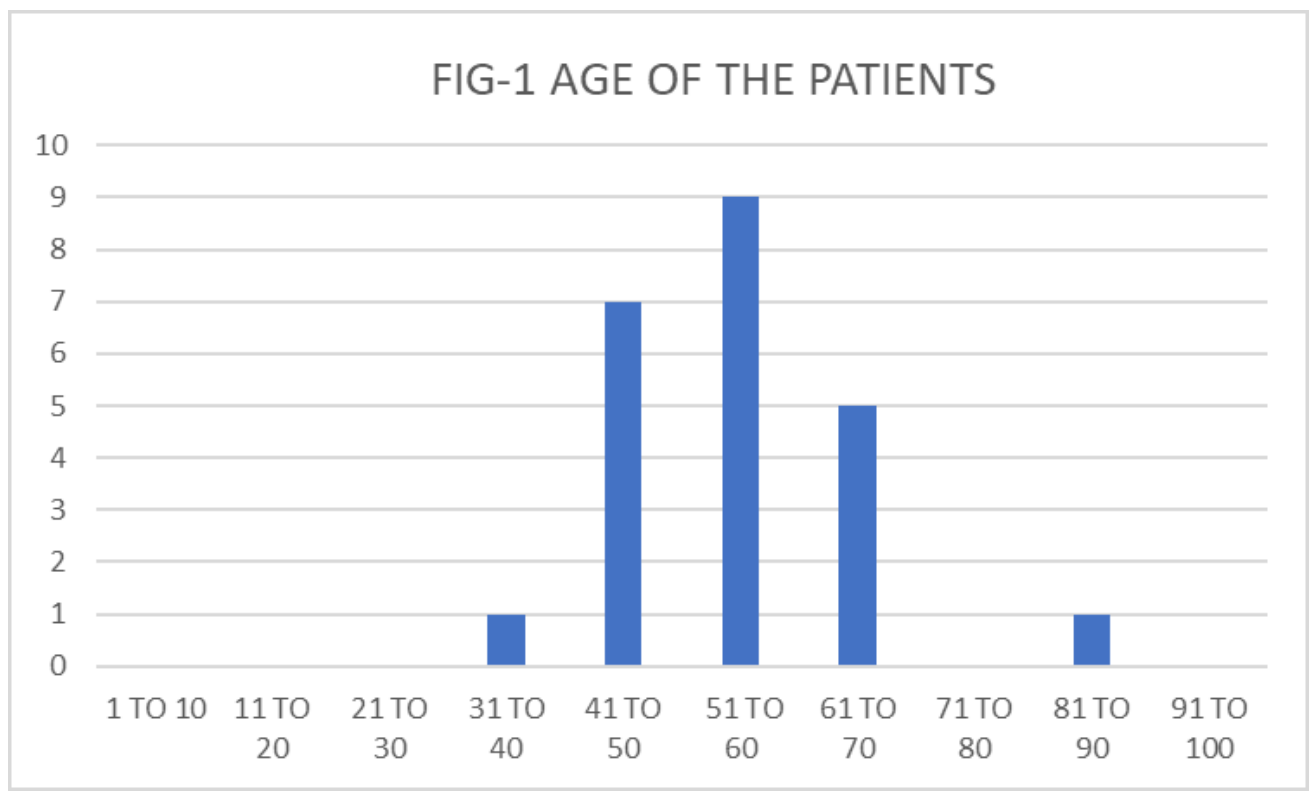

Chart.2 \& 3 Socioeconomic status and Literacy of the study population $(n=23)$
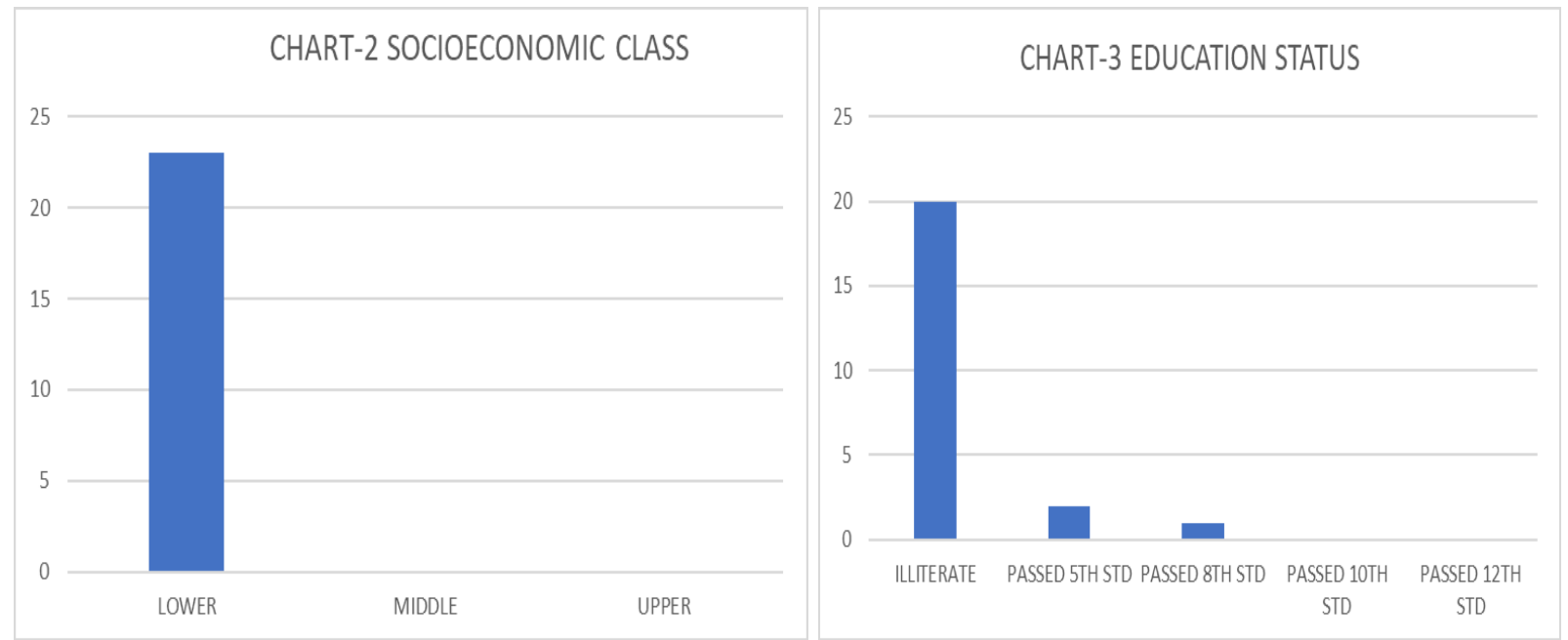
Chart.4 and 5 Marital age \& age of first child birth
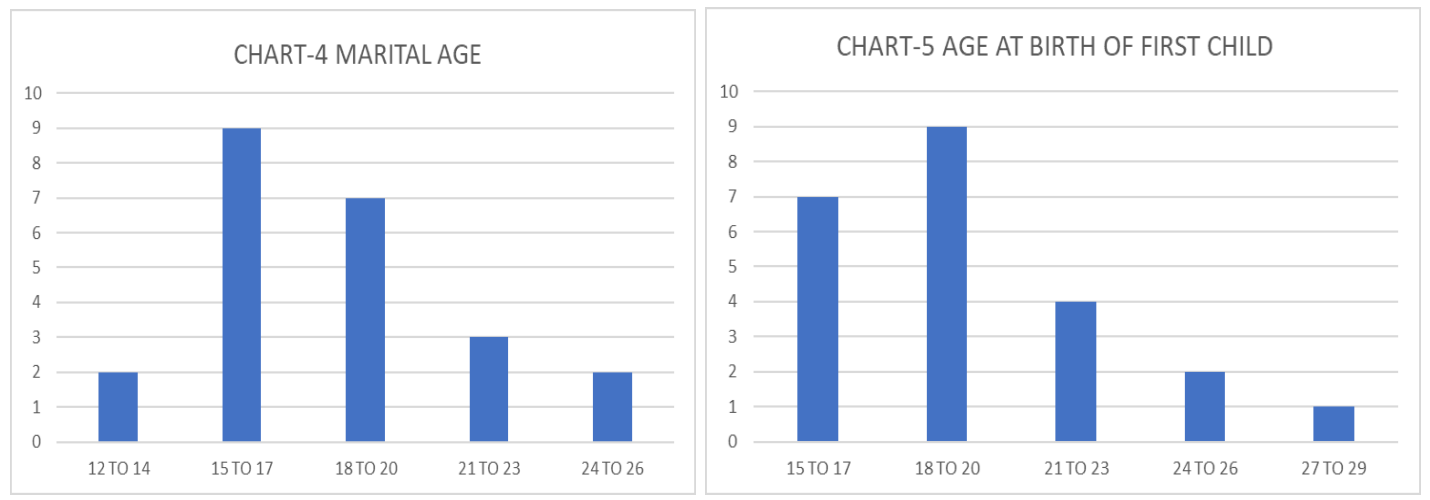

Chart.6 Parity of the study population $(n=23)$

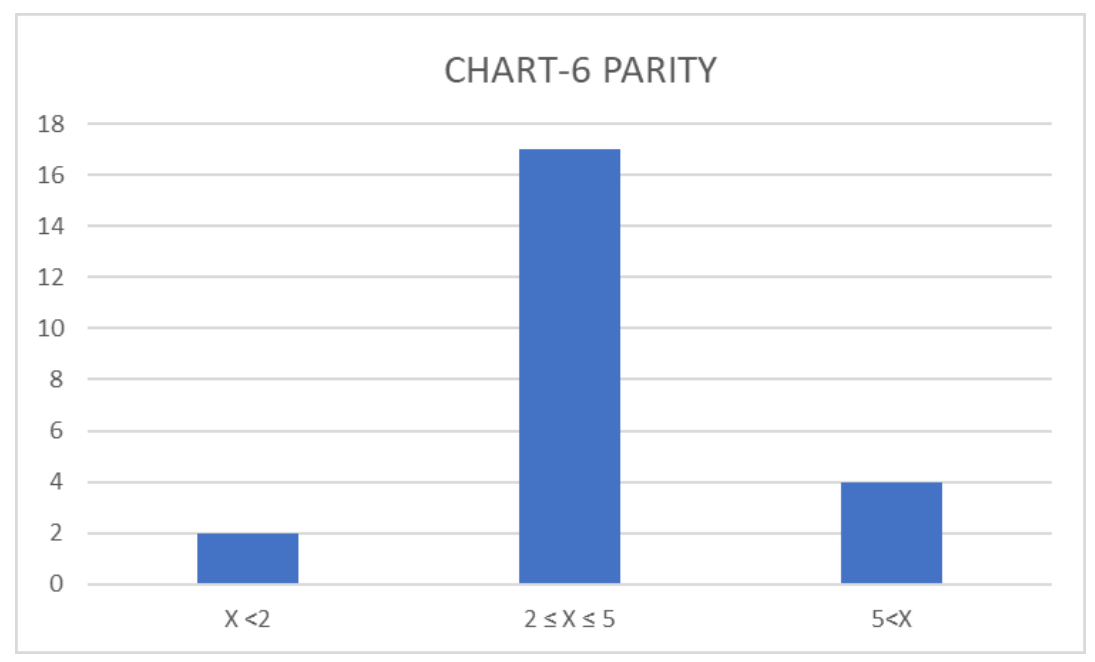

Chart.7 Sanitary conditions of the study population $(n=23)$

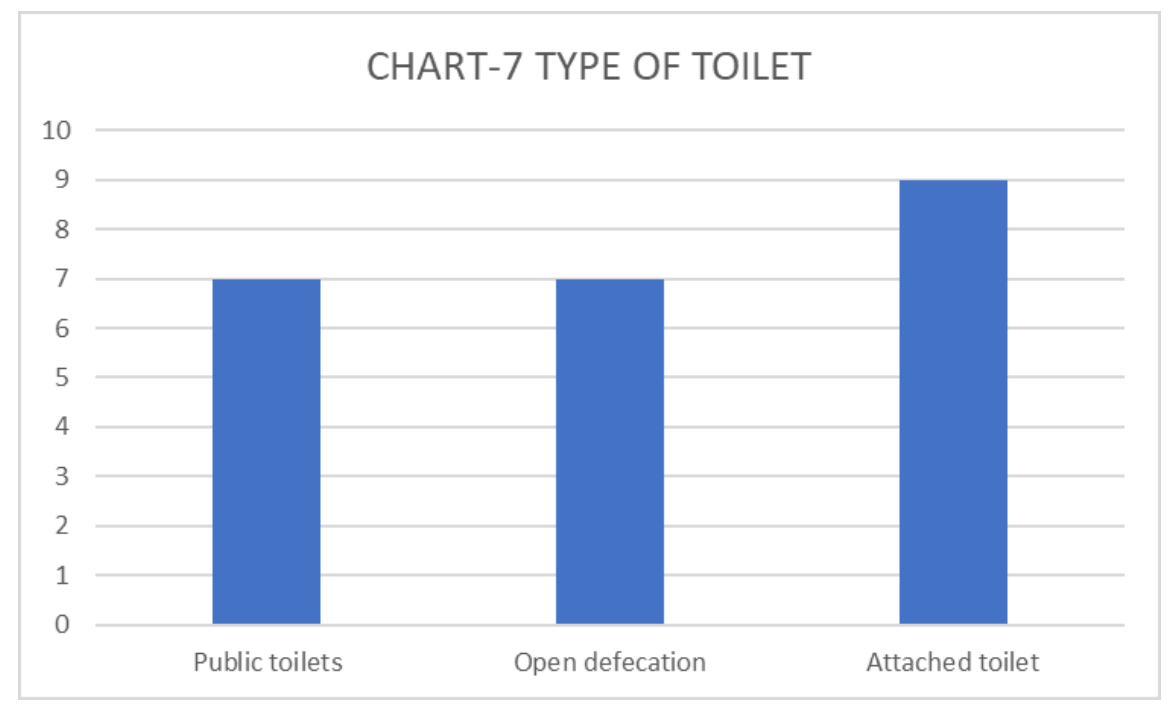


Chart.8 Associated immunosuppressed condition $(n=23)$

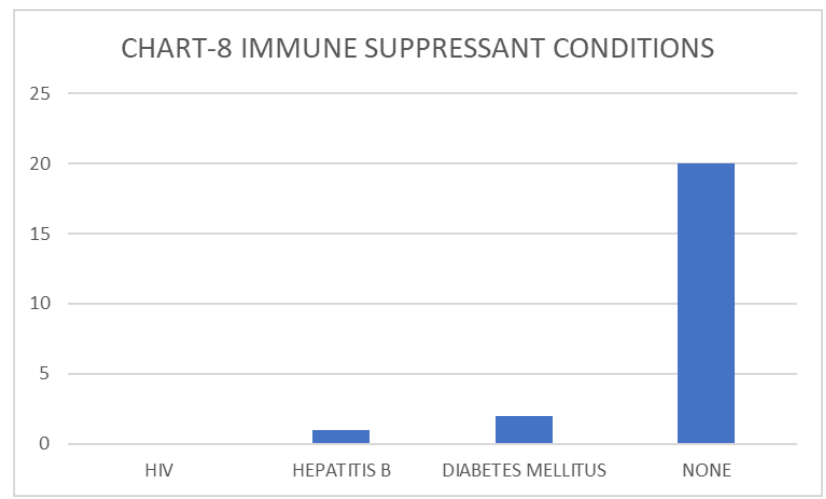

Chart.9 Clinical stage \& type of cancer $(n=23)$
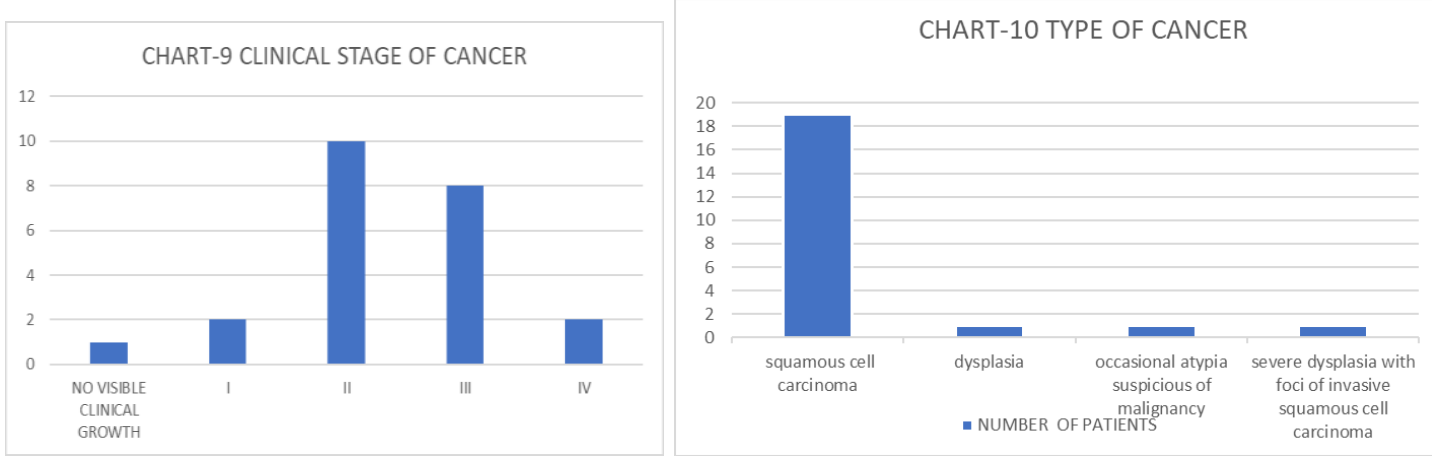

Chart.10 Strain of HPV associated with the tumors $(n=23)$

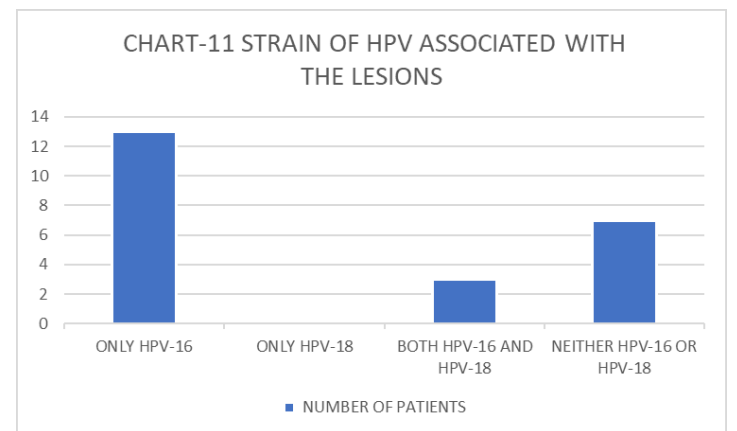

This pattern is similar to that observed by Bosch et al., (1995) who stated that HPV strain 16 was the most prevalent strain among cancers of the cervix worldwide. It also concurs with Basu et al., (2011) and Berlin Grace et al., (2009) who have noted the preponderance of HPV strain 16 among Indian, and more specifically, Chennai populations respectively.
Our finding of HPV strains 16 and/or 18 in $69.5 \%$ cases is consistent with the data of $\mathrm{Ni}$ Li et al., (2011) which showed that in Eastern Asia, $63 \%$ of invasive cervical cancers (ICC) were associated with these strains.

The association of HPV strains with specific histopathologic forms of CA cervix also mirrors the experience of other groups like 
Clifford et al., (2003). We too found a high association (69.5\%) between strain 16 and squamous cell carcinoma of the cervix. The pattern of association of specific high-risk HPV strains with CA cervix in our region has implications for the choice of HPV vaccine to safeguard young women against developing HPV infections that might lead on to malignancy.

The quadrivalent vaccine, which is preferred over the monovalent HPV-16 vaccine due to its higher efficacy (Markowitz LE et al., 2007), protects against 2 high-risk HPV strains (16 and 18), and two low-risk HPV strains (6 and 11). If administered prophylactically, this vaccine would have prevented $70 \%$ of the observed cases in our sample.

However, the narrow specificity of the vaccine means that it lacks meaningful cross protection against other HPV strains. Therefore, the absence of either high-risk strain covered by the vaccine (strains 16 and 18) in $30.5 \%$ of our population is cause for concern and needs further evaluation.

A nonavalent HPV vaccine, covering $9 \mathrm{HPV}$ strains including 31, 33, 45, 52, and 58 in addition to those covered under the quadrivalent vaccine, is now available. But in most vaccination programs, the quadrivalent vaccine is still preferred for economic reasons.

If further study demonstrates the presence of one of the additional five HPV strains in our $30.5 \%$ patients who were negative for HPV strains 16 and 18, then there might be a strong case to support the move to a nonavalent HPV vaccine for protection of the community because the immunity provided by these vaccines is lifelong and the cost of vaccine prophylaxis is much lower than the expense of treating established disease.
A simple quadrivalent vaccine would protect most of the population. But there still remains a significant segment of the population who might be exposed to other high-risk strains despite vaccination.

A limitation of our study, apart from its small sample size, is that we were unable to screen for other high-risk HPV strains that may have been associated with CA cervix in the 7 patients who were negative for strains 16 and 18.

In addition to measures that counter known risk factors for CA cervix, such as providing wider access to properly maintained toilets, educating the public about necessity for basic hygiene, informing high-risk groups about the dangers of sexual promiscuity, high parity and multiple partners, and encouraging young women to undergo regular periodic screening, a well-planned and delivered HPV vaccination program that protects against high-risk HPV strains prevalent in the region might eradicate the problem forever.

This study demonstrates the clear association of HPV infection with cervical carcinomas. There was a high correlation between the high-risk HPV strain 16 and invasive cervical carcinoma. The presence of high risk HPV strains 16 and 18 in $69.5 \%$ of the sample suggests that vaccine prophylaxis would significantly reduce the incidence of $\mathrm{CA}$ cervix.

The fact that $30.5 \%$ of our cases had neither HPV strains 16 or 18 suggests the need for further analysis to see if any other high-risk HPV strains are associated with cervical cancer in our geographic area. This information may guide the choice of an appropriate HPV vaccine for protection of the community.

General measures to improve hygiene and awareness, and regular cancer screening 
programs can pick up cases at an early stage and influence known risk factors for HPV infection and CA cervix, strengthening the positive impact of HPV vaccination on community health enhancement.

\section{Acknowledgement}

We hereby thank the faculty of King Institute of Preventive Medicine \& Research, Guindy, Chennai for giving technical support.

\section{References}

Ahmadi S, Goudarzi H, Jalilvand A, Esmaeilzadeh A. Human Papilloma Virus Genotype Distribution in Cervical lesions in Zanjan, Iran. Asian Pac J Cancer Prev. 2017 Dec 29; 18(12): 3373-3377

Barbosa MS, Schlegel R. The E6 and E7 genes of HPV-18 are sufficient for inducing two stage in vitro transformation of human keratinocytes. Oncogene. 1989 Dec; 4(12): 1529-32.

Basu P, Chandna P, Bamezai RN, Siddiqi M, Saranath D, Lear A, et al., Mass array spectrometry is more sensitive than PreTect HPV-Proofer and consensus PCR for type specific detection of highrisk oncogenic human papilloma virus genotypes in cervical cancer. J Clin Microbiol. 2011 Oct; 49(10):3537-44.

Bedoya-Pilozo CH, Medina Magues LG, Espinosa-Garcia M, Sanchez M, Parrales Valdiviezo JV, Molina D, et al., Molecular epidemiology and phylogenetic analysis of human papillomavirus infection in women with cervical lesions and cancer from the coastal region of Ecuador. Rev Argent Microbiol. 2017 Nov 17; S03257541(17)30137-2

Berlin Grace VM. HPV type 18 is more oncopotent than HPV16 in uterine cervical carcinogenesis although
HPV16 is the prevalent type in Chennai, India. Indian J Cancer. 2009 Jul-Sep; 46(3):203-7

Bosch FX, Lorincz A, Munoz N, Meijer CJ, Shah KV. The causal relation between human papillomavirus and cervical cancer. J Clin Pathol. 2002 Apr; 55(4):244-65.

Bosch FX, Manos M, Munoz N, Sherman M, Jansen AM, Peto J, et al., Prevalence of human papillomavirus in cervical cancer: A worldwide perspective. J Natl Cancer Inst. 1995 Jun 7; 87(11):796802

Boyer SN, Wazer DE, Band V. E7 protein of human papilloma virus-16 induces degradation of retinoblastoma protein through the ubiquitin-proteasome pathway. Cancer Res. 1996 Oct 15; 56(20):4620-4.

Burger RA, Monk BJ, Kurosaki T. Human papillomavirus type 18: association with poor prognosis in early stage cervical cancer. J Natl Cancer Inst. 1996 Oct 2; 88(19):1361-8.

Clifford GM, Smith JS, Aguado T, Franceschi S. Comparison of HPV type distribution in high-grade cervical lesions and cervical cancer: a meta-analysis. $\mathrm{Br} \mathrm{J}$ Cancer. 2003 Jul 7; 89(1):101-5.

Clifford GM, Smith JS, Plummer M, Munoz N, Franceschi S. Human papillomavirus types in invasive cervical cancer worldwide: a meta-analysis. $\mathrm{Br} \mathrm{J}$ Cancer. 2003 Jan 13; 88(1):63-73.

Faridi R, Zahra A, Khan K, Idrees M. Oncogenic potential of Human Papillomavirus (HPV) and its relation with cervical cancer. Virology J 2011 Jun 3; 8:269.

Li N, Franceschi S, Howell-Jones R, Snijders PJ, Clifford GM. Human papillomavirus type distribution in 30,848 invasive cervical cancers worldwide: Variation by geographical region, histological type and year of publication. Int $\mathbf{J}$ 
Cancer. 2011 Feb 15; 128(4):927-35.

Mantovani F, Banks L. The human papillomavirus E6 protein and its contribution to malignant progression. Oncogene. 2001 Nov 26; 20(54):787487.

Markowitz LE, Dunne EF, Saraiya M, Lawson HW, Chesson H, Unger ER, et al., Quadrivalent Human Papillomavirus VaccineRecommendations of the Advisory Committee on Immunization Practices (ACIP). MMWR Recomm Rep. 2007 Mar 23; 56(RR-2):1-24

Muliira RS, Salas AS, O'Brien B. Quality of Life among Female Cancer Survivors in Africa: An Integrative Literature Review. Asia Pac J Oncol Nurs. 2017 Jan-Mar; 4(1): 6-17.

Munoz N, Bosch FX, de Sanjose S, Herrero $\mathrm{R}$, Castellsague X, Shah KV, et al., Epidemiologic classification of human papillomavirus types associated with cervical cancer. N Engl J Med. 2003 Feb 6; 348(6):518-27.

Nakagawa S, Yoshikawa H, Onda T, Kawana
T, Iwamoto A, Taketani Y. Type of human papillomavirus is related to clinical features of cervical carcinoma. Cancer. 1996 Nov 1; 78: 1935-41

National Cancer Institute. 2014-03-14. "Cervical Cancer Treatment (PDQ®)". Archived from the original on 5 July 2014.

Schiller JT, Day PM, and Kines RC. Current understanding of the mechanism of HPV infection. Gynecol Oncol. 2010 Jun; 118 (1 Suppl): S12-S17.

Sreedevi A, Javed R, Dinesh A. Epidemiology of cervical cancer with special focus on India. Int $\mathrm{J}$ Womens Health. 2015 Apr 16; 7:405-14

Walboomers JM, Jacobs MV, Manos M, Bosch FX, Kummer JA, Shah KV, et al., Human papillomavirus is a necessary cause of invasive cervical cancer worldwide. J. Pathol. 1999; 189: 12-9

World Cancer Report 2014. World Health Organization. 2014. pp. Chapter 5.12.

\section{How to cite this article:}

Radhika Katragadda, S. Ravikumar and Kirtana, S. 2019. Human Papilloma Virus in Cervical Cancer - An Institutional Study. Int.J.Curr.Microbiol.App.Sci. 8(06): 1556-1565. doi: https://doi.org/10.20546/ijcmas.2019.806.187 